\title{
P. ERDÖS
}

J.-L. NICOLAS

\section{A. SÁRKOZY}

\section{Sommes de sous-ensembles}

Séminaire de Théorie des Nombres de Bordeaux, tome 3, $\mathrm{n}^{\circ} 1$ (1991), p. 55-72

<http://www.numdam.org/item?id=JTNB_1991_3_1_55_0>

(C) Université Bordeaux 1, 1991, tous droits réservés.

L'accès aux archives de la revue «Séminaire de Théorie des Nombres de Bordeaux » (http://jtnb.cedram.org/) implique l'accord avec les conditions générales d'utilisation (http://www.numdam.org/conditions). Toute utilisation commerciale ou impression systématique est constitutive d'une infraction pénale. Toute copie ou impression de ce fichier doit contenir la présente mention de copyright.

\section{Numdam}

Article numérisé dans le cadre du programme

Numérisation de documents anciens mathématiques

http://www.numdam.org/ 
Séminaire de Théorie des Nombres,

Bordeaux 3 (1991), 55-72

\title{
Sommes de sous-ensembles.
}

\author{
par P. ERDÖS, J.-L. NICOLAS, A. SÁRKOZY(1)
}

\begin{abstract}
Résumé - On dit qu'un ensemble $\mathcal{A}$ est admissible si les sommes des éléments de deux sous ensembles de $\mathcal{A}$ de cardinaux différents sont différentes. Nous démontrons que si $\mathcal{A} \subset\{1,2, \ldots, N\}$ est admissible, alors Card $A \leq(1+o(1))(143 / 27)^{1 / 2} \sqrt{N}$, améliorant ainsi les résultats de Erdös et Straus, et nous formulons quelques conjectures d'après des calculs numériques. Enfin nous construisons un ensemble infini admissible $\mathcal{A}$ vérifiant $A(x)=\operatorname{Card}\{a \in \mathcal{A} ; a \leq x\}>>x^{5-2 \sqrt{6}}$.

Abstract - $A$ set $\mathcal{A}$ is said to be admissible if the sums of the elements of two subsets of $\mathcal{A}$ of different cardinalities are different. We shall prove that if $\mathcal{A} \subset\{1,2, \ldots, N\}$ is an admissible set, then Card $\mathcal{A} \leq$ $(1+o(1))(143 / 127)^{1 / 2} \sqrt{N}$ improving preceding results of Erdös and Straus. From numerical calculations, some conjectures are given. Finally, we construct an infinite admissible set $\mathcal{A}$ such that $A(x)=\operatorname{Card}\{a \in \mathcal{A} ; a \leq$ $x\}>x^{5-2 \sqrt{6}}$.
\end{abstract}

\section{Introduction}

Tout au long de cet article nous utiliserons les notations suivantes : nous écrirons $\mathbb{N}=\{1,2,3, \ldots\}$ et $\mathbb{N}_{M}=\{1,2, \ldots, M\}$. Nous dirons qu'un ensemble $\mathcal{A} \subset \mathbb{N}$ est admissible, si les sommes des éléments de deux sous ensembles de $\mathcal{A}$ de cardinaux différents sont différentes. Cette définition a été introduite par E.G. Straus ([2]). Si $\mathcal{A} \subset \mathbb{N}$ et $k \in \mathbb{N}$, nous désignerons par $|\mathcal{A}|$ le cardinal de $\mathcal{A}$ et par $\mathcal{P}(\mathcal{A}, k)$ l'ensemble des entiers qui peuvent être représentés comme la somme d'exactement $k$ éléments distincts de $\mathcal{A}$, et nous écrirons $P(\mathcal{A}, k)=|\mathcal{P}(\mathcal{A}, k)|$. Dire que $\mathcal{A}$ est admissible équivaut à dire que

$$
k \neq \ell \Rightarrow P(\mathcal{A}, k) \cap \mathcal{P}(\mathcal{A}, \ell)=\emptyset .
$$

En d'autres termes, $\mathcal{A}$ est admissible si la somme des éléments d'une partie de $\mathcal{A}$ détermine le cardinal de cette partie.

Manuscrit reçu le 30 octobre 1990.

(1) Recherche partiellement financée par la Fondation Nationale Hongroise pour la Recherche Scientifique, contrat $n^{\circ} 1811$ et par le C.N.R.S. SDI 5614 et PRC Mathématiques-Informatique. 
P. Erdös ([1]) a posé et étudié le problème suivant : Quel est le cardinal maximum $F(N)$ d'une partie admissible $\mathcal{A}$ de $\mathbb{N}_{N}$ ? Ses résultats ont été améliorés par E.G. Straus ([2]), qui a démontré :

$$
\text { (i)(1) } \quad \limsup _{N \rightarrow \infty} F(N) N^{-1 / 2} \leq 4 / \sqrt{3} \quad(=2.309401 \ldots) .
$$

De plus, Erdös a conjecturé que le cardinal maximum $F(N)$ est atteint lorsque $\mathcal{A}$ est formé d'entiers consécutifs incluant $N$, c'est-à-dire

$$
\mathcal{A}=\{N-F(N)+i ; 1 \leq i \leq F(N)\} .
$$

En rapport avec cette conjecture, Straus a démontré :

(ii) L'ensemble $\mathcal{A}=\{N-k+1, N-k+2, \ldots, N\}$ est admissible pour $k=2 m-1$ si $m^{2} \leq N<m^{2}+m$ et pour $k=2 m$ si $m^{2}+m \leq N<(m+1)^{2}$.

Ceci implique :

$$
\liminf _{N \rightarrow+\infty} F(N) N^{-1 / 2} \geq 2 .
$$

Nous nous proposons dans cet article d'étendre et de préciser les résultats de Erdős et Straus. D'abord, nous améliorons la borne supérieure de (i). Ensuite, au paragraphe 4, à l'aide des tables numériques construites par J.-P. Massias et M. Deléglise, que nous avons plaisir à remercier ici, nous ferons des conjectures sur ce problème. Finalement, nous étudierons les ensembles infinis admissibles, dans le cinquième paragraphe.

2. THÉORÈME 1. On a :

$$
\limsup _{N \rightarrow \infty} F(N) N^{-1 / 2} \leq(143 / 27)^{1 / 2} \quad(=2.301368 \ldots) .
$$

Il serait possible d'améliorer ce résultat par une application plus élaborée de nos idées. Cependant, il semble impossible d'obtenir de cette façon la limite conjecturée : $\limsup F(N) N^{-1 / 2}=2$ et en fait, une nouvelle idée semble nécessaire pour donner une borne supérieure plus petite que 2.2.

Pour démontrer le théorème 1 , nous avons besoin de deux lemmes.

Lemme 1. Soit $\mathcal{A}$ un ensemble fini, et $k \in \mathbb{N}$ vérifiant $k \leq|\mathcal{A}|$. Alors on a :

$$
P(\mathcal{A}, k) \geq k(|\mathcal{A}|-k)+1 .
$$

Démonstration. C'est le théorème 2 de Straus [2], qui se démontre facilement par récurrence sur $|\mathcal{A}|$.

Nous utiliserons le théorème 4 de Straus sous la forme suivante : 
Lemme 2. Soit $N \in \mathbb{N}$. On a :

$$
F(N)<\frac{4}{\sqrt{3}} N^{1 / 2}+1
$$

Démonstration. Nous suivons la preuve de Straus. Soit $\mathcal{A} \subset \mathbb{N}_{N}$ un ensemble admissible. Il est clair que les éléments de $\mathcal{P}(\mathcal{A}, k)$ sont inférieurs à $k N$. Donc, pour $x \geq 1$, nous avons par le lemme 1 :

$$
\begin{aligned}
x N & \geq\left|\bigcup_{k=1}^{x} \mathcal{P}(\mathcal{A}, k)\right|=\sum_{k=1}^{x} P(\mathcal{A}, k) \\
& \geq \sum_{k=1}^{x}(k(|\mathcal{A}|-k)+1)=|\mathcal{A}| \frac{x(x+1)}{2}-\frac{x(x+1)(2 x+1)}{6}+x
\end{aligned}
$$

d'où il vient, en posant $x=[3|\mathcal{A}| / 4]$ (la notation [u] désigne la partie entière de u)

$$
\begin{aligned}
6(N-1) & \geq 3|\mathcal{A}|(x+1)-(x+1)(2 x+1) \\
& >3|\mathcal{A}| \cdot \frac{3|\mathcal{A}|}{4}-\left(3 \frac{|\mathcal{A}|}{4}+1\right)\left(2 \frac{3|\mathcal{A}|}{4}+1\right) \\
& =\frac{9}{8}|\mathcal{A}|^{2}-\frac{9}{4}|\mathcal{A}|-1=\frac{9}{8}|\mathcal{A}|(|\mathcal{A}|-2)-1 .
\end{aligned}
$$

Supposons maintenant que l'on ait :

$$
|\mathcal{A}| \geq \frac{4}{\sqrt{3}} N^{1 / 2}+1
$$

contrairement à (4). Cela impliquerait

$$
\begin{aligned}
\frac{9}{8}|\mathcal{A}|(|\mathcal{A}|-2)-1 \geq \frac{9}{8}\left(\frac{4}{\sqrt{3}} N^{1 / 2}+1\right)\left(\frac{4}{\sqrt{3}} N^{1 / 2}-1\right)-1 & \\
= & 6 N-\frac{9}{8}-1>6(N-1)
\end{aligned}
$$

ce qui contredit (5), et achève la preuve du lemme 2. 


\section{Démonstration du Théorème 1.}

Nous distinguerons 3 cas. Soit $\mathcal{A}=\left\{a_{1}, a_{2}, \ldots, a_{y}\right\} \subset \mathbb{N}_{N},\left(a_{1}<a_{2}<\right.$ $\left.\ldots<a_{y}\right)$ un ensemble admissible. On pose :

$$
\begin{aligned}
\mathcal{A}_{1} & =\{a ; a \in \mathcal{A}, a \leq N / 2\} \\
\mathcal{A}_{2} & =\{a ; a \in \mathcal{A}, N / 2<a \leq 17 N / 18\} \\
\text { et } \quad \mathcal{A}_{3} & =\{a ; a \in \mathcal{A}, 17 N / 18<a\}
\end{aligned}
$$

PREMIER CAS. On suppose que

$$
\left|\mathcal{A}_{3}\right|<\frac{21}{32} y=\frac{21}{32}|\mathcal{A}| \text {. }
$$

Comme dans la démonstration du lemme 2, nous partons de la relation $\left|\bigcup_{k=1}^{x} \mathcal{P}(\mathcal{A}, k)\right|=\sum_{k=1}^{x} P(\mathcal{A}, k)$ avec toujours $x=[3 y / 4]$. Le plus grand élément de $\bigcup_{k=1}^{x} \mathcal{P}(\mathcal{A}, k)$ est évidemment le plus grand élément de $\mathcal{P}(\mathcal{A}, x)$, et $\operatorname{par}(6)$, il vaut

$$
\begin{aligned}
& \sum_{j=0}^{x-1} a_{y-j} \leq \sum_{a \in \mathcal{A}_{3}} a+\left(x-\left|\mathcal{A}_{3}\right|\right) \frac{17 N}{18} \\
& \leq\left|\mathcal{A}_{3}\right| N+\left(x-\left|\mathcal{A}_{3}\right|\right) \frac{17 N}{18}=\left|\mathcal{A}_{3}\right| \frac{N}{18}+x \frac{17 N}{18} \\
& <\frac{21}{32} y \frac{N}{18}+\frac{17}{18} x N=\frac{3}{4} y \frac{7}{144} N+\frac{17}{18} x N \\
& <(x+1) \frac{7}{144} N+\frac{17}{18} x N<\frac{143}{144} x N+\frac{N}{20}
\end{aligned}
$$

Nous avons donc :

$$
\sum_{k=1}^{x} P(\mathcal{A}, k)=\left|\bigcup_{k=1}^{x} \mathcal{P}(\mathcal{A}, k)\right|<\frac{143}{144} x N+\frac{N}{20} .
$$

Par le lemme 1, ceci entraîne :

$$
\begin{aligned}
\frac{143}{144} x N+ & \frac{N}{20}>\sum_{k=1}^{x} P(\mathcal{A}, k) \geq \sum_{k=1}^{x}(k(y-k)+1) \\
& =y \sum_{k=1}^{x} k-\sum_{k=1}^{x} k^{2}+x=y \frac{x(x+1)}{2}-\frac{x(x+1)(2 x+1)}{6}+x
\end{aligned}
$$


et en utilisant $x=[3 y / 4]$,

$$
\frac{143}{144} \frac{3}{4} y N+\mathrm{O}(N)>\frac{1}{2}\left(\frac{3}{4}\right)^{2} y^{3}-\frac{1}{3}\left(\frac{3}{4}\right)^{3} y^{3}+\mathrm{O}\left(y^{2}\right) .
$$

Nous avons donc, lorsque $y \rightarrow \infty$,

$$
\begin{aligned}
& y^{2}<(1+o(1)) \frac{143}{192}\left(\frac{1}{2}\left(\frac{3}{4}\right)^{2}-\frac{1}{3}\left(\frac{3}{4}\right)^{3}\right)^{-1} N=\left((1+o(1)) \frac{143}{27} N\right. \\
& \text { et pour } N \rightarrow+\infty
\end{aligned}
$$

$$
y<(1+o(1))\left(\frac{143}{27} N\right)^{1 / 2}
$$

\section{DEUXIÈME CAS.}

On suppose que

$$
\left|\mathcal{A}_{3}\right| \geq \frac{21}{32} y
$$

et

$$
\left|\mathcal{A}_{1}\right|<\frac{1}{32} y
$$

Posons $z=\left[\frac{3}{32} y\right]+1$. Le plus petit élément de $\bigcup_{k=z}^{x} \mathcal{P}(\mathcal{A}, k)$ est le plus petit élément de $\mathcal{P}(\mathcal{A}, z)$ et vaut :

$$
\begin{aligned}
\sum_{j=1}^{z} a_{j} & =\sum_{j=1}^{\left|\mathcal{A}_{1}\right|} a_{j}+\sum_{j=\left|\mathcal{A}_{1}\right|+1}^{z} a_{j}>\sum_{j=\left|\mathcal{A}_{1}\right|+1}^{z} N / 2 \\
= & \left(z-\mid \mathcal{A}_{1}\right) \frac{N}{2}>\left(\frac{3}{32} y-\frac{1}{32} y\right) \frac{N}{2}=\frac{y N}{32},
\end{aligned}
$$

en utilisant (9). Par ailleurs, le plus grand élément de $\bigcup_{k=z}^{x} \mathcal{P}(\mathcal{A}, k)$ est

$$
\sum_{j=0}^{x-1} a_{y-j} \leq \sum_{j=0}^{x-1} N=x N \leq \frac{3}{4} y N,
$$

et donc

$$
\bigcup_{k=z}^{x} \mathcal{P}(\mathcal{A}, k) \subset\left[\frac{y N}{32}, \frac{3}{4} y N\right]
$$


Puisque $\mathcal{A}$ est admissible, ceci entraîne,

$$
\sum_{k=z}^{x} P(\mathcal{A}, k)=\left|\bigcup_{k=z}^{x} \mathcal{P}(\mathcal{A}, k)\right|<\frac{3}{4} y N-\frac{1}{32} y N+1
$$

$$
=\frac{23}{32} y N+1
$$

Maintenant, par le lemme 1, nous obtenons la minoration :

$$
\begin{array}{r}
\sum_{k=z}^{x} P(\mathcal{A}, k) \geq \sum_{k=z}^{x}(k(y-k)+1)=y \sum_{k=z}^{x} k-\sum_{k=z}^{x} k^{2}+x-z+1 \\
=y \frac{x(x+1)-(z-1) z}{2}-\frac{x(x+1)(2 x+1)}{6}+\frac{(z-1) z(2 z-1)}{6}+x-z+1 \\
=\frac{1}{2} y\left(\left(\frac{3}{4} y\right)^{2}-\left(\frac{3}{32} y\right)^{2}\right)-\frac{1}{3}\left(\frac{3}{4} y\right)^{3}+\frac{1}{3}\left(\frac{3}{32} y\right)^{3}+\mathrm{O}\left(y^{2}\right) \\
=\frac{4473}{32768} y^{3}+\mathrm{O}\left(y^{2}\right) .
\end{array}
$$

On déduit de (10) et (11) que :

$$
\frac{23}{32} N>\frac{4473}{32768} y^{2}+\mathrm{O}(y)
$$

d'où il vient lorsque $N \rightarrow \infty$,

$$
y<(1+o(1))\left(\frac{23552}{4473} N\right)^{1 / 2}\left(=(1+o(1)) 2.294639 \ldots N^{1 / 2}\right) .
$$

TROISIÈME CAS. Nous supposons maintenant

$$
\left|\mathcal{A}_{3}\right| \geq \frac{21}{32} y
$$

et

$$
\left|\mathcal{A}_{1}\right| \geq \frac{1}{32} y
$$

Posons $u=[y / 32]$. Nous allons d'abord minorer $P(\mathcal{A}, k)$ lorsque

$$
u \leq k<9 u
$$


Considérons toutes les sommes de la forme

$$
a_{1}+a_{2}+\ldots+a_{u}+a_{i_{1}}+a_{i_{2}}+\ldots+a_{i_{k-n}}
$$

avec

$a_{i_{1}}<a_{i_{2}}<\ldots<a_{i_{k}-u}$, et $a_{i_{j}} \in \mathcal{A}_{3}$ pour $j=1,2, \ldots, k-u$. Le nombre de sommes de cette forme est $P\left(\mathcal{A}_{3}, k-u\right)$ et par (14) et (15) chacune de ces sommes ne dépasse pas

$$
u \frac{N}{2}+(k-u) N=\left(k-\frac{u}{2}\right) N<\left(k-\frac{k}{18}\right) N=\frac{17}{18} k N .
$$

De plus, pour

$$
a_{j_{1}}+a_{j_{2}}+\ldots+a_{j_{k}} \in \mathcal{P}\left(\mathcal{A}_{3}, k\right)
$$

nous avons

$$
a_{j_{1}}+a_{j_{2}}+\ldots+a_{j_{k}}>k \frac{17 N}{18}=\frac{17}{18} k N
$$

et les sommes dans (17) sont ainsi plus grandes que les sommes dans (16). Par ailleurs, les sommes dans (16) et dans (17) appartiennent à $\mathcal{P}(\mathcal{A}, k)$. Ainsi, $P(\mathcal{A}, k)$ est supérieur ou égal au nombre total des sommes dans (16) et dans (17) :

$$
P(\mathcal{A}, k) \geq P\left(\mathcal{A}_{3}, k-u\right)+P\left(\mathcal{A}_{3}, k\right) \quad(\text { pour } u \leq k<9 u) .
$$

D'où il vient par (13) et le lemme 1 :

$$
\begin{aligned}
P(\mathcal{A}, k) & \geq(k-u)\left(\left|\mathcal{A}_{3}\right|-(k-u)\right)+1+k\left(\left|\mathcal{A}_{3}\right|-k\right)+1 \\
& =(2 k-u)\left|\mathcal{A}_{3}\right|-2 k^{2}+2 k u-u^{2}+2 \\
& >\left(2 k-\frac{y}{32}\right) \frac{21}{32} y-2 k^{2}+2 k\left(\frac{y}{32}-1\right)-\left(\frac{y}{32}\right)^{2} \\
& =\left(\frac{11}{8} y-2\right) k-\frac{11}{512} y^{2}-2 k^{2} \quad(\text { pour } u \leq k<9 u) .
\end{aligned}
$$

Le plus grand élément de $\bigcup_{k=1}^{x} \mathcal{P}(\mathcal{A}, k)$ est $\leq x N$ (où, comme précédemment, $x=[3 y / 4])$, et comme $\mathcal{A}$ est admissible,

$$
\sum_{k=1}^{x} P(\mathcal{A}, k) \leq x N
$$


Par le lemme 1 et (18) nous avons :

$$
\begin{aligned}
& \frac{3}{4} y N \geq x N \geq \sum_{k=1}^{x}(k(y-k)+1) \\
& +\sum_{k=u}^{9 u-1}\left(\left(\left(\frac{11}{8} y-2\right) k-\frac{11}{512} y^{2}-2 k^{2}\right)-k(y-k)-1\right) \\
& =\sum_{k=1}^{[3 y / 4]}(k(y-k)+1)+\sum_{k=u}^{9 u-1}\left(\left(\frac{3}{8} y-2\right) k-\frac{11}{512} y^{2}-k^{2}-1\right) \\
& =y \sum_{k=1}^{[3 y / 4]} k-\sum_{k=1}^{[3 y / 4]} k^{2}+\mathrm{O}(y)+\frac{3}{8} y \sum_{k=u}^{9 u-1} k-\frac{11}{512} y^{2} \cdot 8 u-\sum_{k=u}^{9 u-1} k^{2}+\mathrm{O}\left(y^{2}\right) \\
& =\frac{1}{2} y\left(\frac{3 y}{4}\right)^{2}-\frac{1}{3}\left(\frac{3 y}{4}\right)^{3}+\frac{3}{8} y \cdot \frac{1}{2} 80 u^{2}-\frac{11}{64} y^{2} u-\frac{728}{3} u^{3}+\mathrm{O}\left(y^{2}\right) \\
& =\left(\frac{9}{32}-\frac{9}{64}+15 \cdot \frac{1}{32^{2}}-\frac{11}{64} \cdot \frac{1}{32}-\frac{728}{3} \cdot \frac{1}{32^{3}}\right) y^{3}+\mathrm{O}\left(y^{2}\right) \\
& =\frac{1751}{12288} y^{3}+\mathrm{O}\left(y^{2}\right)
\end{aligned}
$$

d'où l'on déduit

$$
y \leq\left((1+\mathrm{o}(1))\left(\frac{9216}{1751} N\right)^{1 / 2}(=1+\mathrm{o}(1)) 2.294183 \ldots N^{1 / 2}\right)
$$

La démonstration du théorème 1 résulte alors de (7), (12) et (19).

\section{Quelques conjectures}

Il est commode de définir :

$$
g(N)=[2 \sqrt{N+1 / 4}-1]
$$

On a donc

$$
\begin{array}{ll}
\text { pour } m^{2} \leq N<m(m+1) & g(N)=2 m-1 \\
\text { pour } m(m+1) \leq N<(m+1)^{2} & g(N)=2 m .
\end{array}
$$

Nous dirons ensuite qu'un ensemble admissible $\mathcal{A}$ est $N$-optimal si $\mathcal{A} \subset \mathbb{N}_{N}$, et $|\mathcal{A}|=F(N)$. Nous noterons $p(N)$ le nombre d'ensembles admissibles 
$N$ optimaux, et par $a(N)$ le nombre d'ensembles admissibles non vides $\mathcal{A} \subset \mathbb{N}_{N}$

Nous dirons aussi qu'un ensemble $\mathcal{A}$ est impair si tous les éléments de $\mathcal{A}$ sont impairs. Dans le cas contraire, c'est-à-dire si $\mathcal{A}$ contient un nombre pair, nous dirons que $\mathcal{A}$ est non impair. Nous définissons

$$
h(N)=\min _{\mathcal{A} \in F_{N}}(\min \mathcal{A})
$$

où $\mathcal{A}$ parcourt l'ensemble $E_{N}$ des ensembles admissibles $N$-optimaux et non impairs. Si $E_{N}=\emptyset$, alors $h(N)=+\infty$.

J.-P. Massias et M. Deléglise ont donné la liste des ensembles $N$-optimaux pour $N \leq 50$, et l'on trouvera un extrait de leurs résultats dans la table en annexe. Ces calculs sur ordinateur montrent un comportement très régulier qui nous conduit aux conjectures suivantes :

Conjecture 1. On a pour tout $N \geq 1$ ]

$$
F(N)=g(N)=[2 \sqrt{N+1 / 4}-1]
$$

et I'ensemble (ii) de Straus est $N$-optimal.

Conjecture 2. Lorsque $N$ est de la forme $m^{2}$ ou $m(m+1)$, on a $p(N)=1$, autrement dit I'ensemble (ii) de Straus est le seul ensemble admissible $N$-optimal. De façon plus générale, soit $t \geq 0$. II existe deux fonctions $m_{0}(t)$ et $q(t)$ telles que, pour $m \geq m_{0}(t)$ on ait

$$
p\left(m^{2}+t\right)=p(m(m+1)+t)=q(t)
$$

et I'on a $q(0)=1, q(1)=2, q(2)=5$.

Conjecture 3. Les ensembles admissibles $N$-optimaux impairs sont :

$\mathcal{A}_{1}=\{N, N-2, \ldots, N-2 g(N)+2\}$ lorsque $N=m^{2}-2$ et $m$ impair, Iorsque $N=m^{2}-1$ et $m$ pair et lorsque $N=m(m+1)-1$ et

$$
\mathcal{A}_{2}=\{N, N-2, \ldots, N-2 g(N)+4, N-2 g(N)\} \text { lorsque } N=m^{2}-1
$$
avec $m$ pair et lorsque $N=m(m+1)-1$.

Il est facile de voir que les ensembles ci-dessus ont $g(N)$ éléments et sont admissibles, et donc sous réserve de la conjecture 1 sont $N$-optimaux. 
De façon plus générale on peut caractériser les progressions arithmétiques admissibles

$$
\mathcal{A}=\mathcal{A}(k, r, N)=\{N, N-r, \ldots, N-(k-1) r\}
$$

avec $r \geq 1, k \geq 1$ et $(N, r)=1 \quad$ par la condition :

$$
(k+r)^{2} \leq 4 N+2 r(r+1)-3 .
$$

Ce résultat se démontre comme (ii) en observant que, comme $(N, r)=1$, il suffit de comparer la somme des $\mathrm{j}$ plus grands termes de $\mathcal{A}$ avec la somme des $(j+r)$ plus petits termes de $\mathcal{A}$, et ceci pour $1 \leq j \leq k-r$.

Un calcul un peu technique montre que pour $r \geq 3, \mathcal{A}(g(N), r, N)$ n'est pas admissible, et pour $r=2$ et $\mathrm{N}$ impair, $\mathcal{A}(g(\bar{N}), 2, N)$ est admissible seulement dans les cas décrits à la conjecture 3 .

Conjecture 4. Pour $N \geq 16$, la fonction $N \mapsto h(N)$ est constante sur les intervalles $\left(m^{2}, m(m+1)-1\right)$ et $\left(m(m+1),(m+1)^{2}-1\right)$. On a de plus $h\left(m^{2}\right)=m^{2}-2 m+2$ et $h(m(m+1))=m^{2}-m+1$.

Comportement de $a(N)$. Il est clair que toute partie d'un ensemble admissible est admissible. Il résulte donc de (ii) que

$$
a(N) \geq 2^{g(N)}-1 \geq 2^{2 \sqrt{N}-2}-1 .
$$

Par ailleurs, il résulte du lemme 2 , en posant $k=\left[\frac{4}{\sqrt{3}} N^{1 / 2}+1\right]$, que

$$
a(N) \leq \sum_{j=0}^{k}\left(\begin{array}{c}
N \\
j
\end{array}\right) \leq N\left(\begin{array}{c}
N \\
k
\end{array}\right) \leq N\left(\frac{N e}{k}\right)^{k} \leq \exp (c \sqrt{N} \log N)
$$

pour un certain $c$.

Il nous semble difficile de préciser davantage le comportement de $a(N)$. L'étude de la table numérique laisse penser que

$$
\log a(N) \asymp \sqrt{N \log N},
$$

mais nous conjecturons cependant que l'on a $\log a(N) \asymp \sqrt{N}$. 


\section{Suites infinies.}

Nous allons maintenant étudier les ensembles infinis admissibles. Si $\mathcal{A} \subset$ $\mathbb{N}$, et $x>0$, on pose

$$
A(x)=\sum_{n \in \mathcal{A}, n \leq x} 1
$$

et nous conjecturons que si $\mathcal{A}$ est admissible, alors

$$
\lim _{x \rightarrow \infty} \inf A(x) x^{-1 / 2}=0
$$

(La limite ci-dessus est finie par (1).) Malheureusement nous n'avons pas été capables de le démontrer.

Par ailleurs, Erdös [1] a démontré qu'il existe $c>0$ (la valeur de $c$ n'est pas précisée) et un ensemble infini admissible $\mathcal{A}$ tel que

$$
A(x)>x^{\circ} \text { pour } x>x_{0}
$$

Peut-être est-il possible d'obtenir

$$
A(x) \gg x^{1 / 2-\epsilon}
$$

Nous prouvons seulement le résultat plus faible :

THÉorème 2. II existe un ensemble infini admissible $\mathcal{A} \subset \mathbb{N}$ vérifiant pour $x>x_{0}$ :

$$
A(x) \gg x^{5-2 \sqrt{6}}\left(=x^{0.10102 \ldots}\right)
$$

Démonstration. Soit $x_{0}=0$, et pour $N=1,2, \ldots$, posons $x_{N}=K^{((2+\sqrt{6}) / 2)^{N}}$, avec $K=40$.

Par récurrence nous allons définir les ensembles de nombres entiers positifs $\mathcal{A}_{1}, \mathcal{A}_{2}, \ldots$ vérifiant

$$
\left.\left.\mathcal{A}_{N} \subset\right] x_{N-1}, x_{N}\right]
$$

Lorsque $N=1$, on pose $T_{1}=1$ et

$$
\mathcal{A}_{1}=\left\{\left[x_{1}\right]-i ; 1 \leq i \leq x_{1}^{(\sqrt{6}-2) / 2}\right\}
$$


Pour $N \geq 2$, supposant construits $\mathcal{A}_{1}, \ldots, \mathcal{A}_{N-1}$, on pose

$$
T_{N}=\sum_{n=1}^{N-1}\left(\sum_{a \in \mathcal{A}_{n}} a\right)+1
$$

et

$$
\mathcal{A}_{N}=\left\{T_{N}\left[x_{N} / T_{N}\right]-i T_{N} ; 1 \leq i \leq x_{N}^{(\sqrt{6}-2) / 2}\right\}
$$

Maintenant nous allons démontrer l'inclusion (21) pour tout N. On remarque d'abord que, pour $N \geq 1$, on a :

$$
x_{N-1} \leq \frac{1}{K} x_{N} \quad \text { et } \quad x_{N} \geq K .
$$

De plus, d'après la définition de $\mathcal{A}_{N}$, on a :

$$
\left.\left.\mathcal{A}_{N} \subset\right] x_{N}-\left(1+x_{N}^{(\sqrt{6}-2) / 2}\right) T_{N}, x_{N}\right]
$$

En tenant compte de (22) et (23), il suffit de démontrer

$$
\left(x_{N}^{(\sqrt{6}-2) / 2}+1\right) T_{N}<\frac{K-1}{K} x_{N}
$$

pour démontrer (21).

Or (24) se vérifie numériquement lorsque $N=1$. Pour $N>1$, les définitions de $\mathcal{A}_{1}, \ldots, \mathcal{A}_{N-1}$ et $T_{N}$ entraînent, par (22)

$$
\begin{aligned}
& T_{N} \leq \sum_{n=1}^{N-1}\left|\mathcal{A}_{n}\right| x_{n}+1 \\
& \leq 1+\sum_{n=1}^{N-1} x_{n}^{(\sqrt{6}-2) / 2} x_{n}=1+\sum_{n=1}^{N-1} x_{n}^{\sqrt{6} / 2} \\
& \leq x_{N-1}^{\sqrt{6} / 2}\left(1+\frac{1}{K}+\ldots+\frac{1}{K^{N-2}}+\frac{1}{x_{N-1}}\right) \leq x_{N-1}^{\sqrt{6} / 2}\left(\frac{K}{K-1}+\frac{1}{K}\right) \\
& \leq \frac{K+1}{K-1} x_{N-1}^{\sqrt{6} / 2}=\frac{K+1}{K-1}\left(x_{N}^{(2 /(2+\sqrt{6})}\right)^{\sqrt{6} / 2}=\frac{K+1}{K-1} x_{N}^{\sqrt{6} /(\sqrt{6}+2)} .
\end{aligned}
$$

Nous avons ainsi

$$
x_{N}^{(\sqrt{6}-2) / 2} T_{N} \leq \frac{K+1}{K-1} x_{N}^{(\sqrt{6}-2) / 2+\sqrt{6} /(\sqrt{6}+2)}
$$




$$
=\frac{K+1}{K-1} x_{N}^{1-1 /(\sqrt{6}+2)} \leq \frac{K+1}{K-1} K^{-1 /(\sqrt{6}+2)} x_{N}
$$

d'où l'on déduit (24) en vérifiant numériquement que

$$
2 \frac{K+1}{K-1} K^{-1 /(\sqrt{6}+2)} \leq \frac{K-1}{K} .
$$

On pose $\mathcal{A}=\bigcup_{N=1}^{\infty} \mathcal{A}_{N}$. Nous allons montrer que cet ensemble est admissible et qu'il vérifie $(20)$.

D'abord nous allons montrer que pour tout $\mathrm{N}$, l'ensemble $\mathcal{A}_{N}$ est admissible. Il suffit pour cela de montrer que pour $1 \leq k \leq\left|\mathcal{A}_{N}\right|-1$,

$$
r \in \mathcal{P}\left(\mathcal{A}_{N}, k\right), s \in \mathcal{P}\left(\mathcal{A}_{N}, k+1\right)
$$

on a

$$
r<s
$$

On pose $y_{N}=T_{N}\left[x_{N} / T_{N}\right]$ et

$$
z_{N}=\left|\mathcal{A}_{N}\right|=\left[x_{N}^{(\sqrt{6}-2) / 2}\right] .
$$

Nous avons alors

$$
\begin{aligned}
s-r & \geq\left(y_{N}-z_{N} T_{N}\right)+\left(y_{N}-\left(z_{N}-1\right) T_{N}\right)+\ldots+\left(y_{N}-\left(z_{N}-k\right) T_{N}\right) \\
& -\left(y_{N}+\left(y_{N}-T_{N}\right)+\ldots+\left(y_{N}-(k-1) T_{N}\right)\right) \\
& =\left(y_{N}-(k+1) z_{N} T_{N}+\left(\frac{k(k+1)}{2}+\frac{(k-1) k}{2}\right) T_{N}\right. \\
& =y_{N}-(k+1) z_{N} T_{N}+k^{2} T_{N}+k^{2} T_{N} \stackrel{\text { def }}{=} f(k) .
\end{aligned}
$$

Le minimum du trinôme $f(k)$ est atteint en $k=z_{N} / 2$ et donc

$$
\begin{aligned}
s-r & \geq f\left(z_{N} / 2\right)=y_{N}-\left(\frac{z_{N}}{2}+1\right) z_{N} T_{N}+\frac{z_{N}^{2}}{4} T_{N} \\
& =y_{N}-\left(\frac{z_{N}^{2}}{4}+z_{N}\right) T_{N} \geq x_{N}-\left(\frac{z_{N}^{2}}{4}+z_{N}+1\right) T_{N} .
\end{aligned}
$$


Par (25) et (28), (29) devient :

$$
\begin{aligned}
s-r & \geq x_{N}-\left(\frac{x_{N}^{\sqrt{6}-2}}{4}+x_{N}^{(\sqrt{6}-2) / 2}+1\right) \frac{K+1}{K-1} x_{N}^{\sqrt{6} /(\sqrt{6}+2)} \\
& =x_{N}\left(1-\frac{K+1}{K-1}\left(\frac{1}{4}+x_{N}^{-1 /(2+\sqrt{6})}+x_{N}^{-2 /(2+\sqrt{6})}\right)\right) \\
& \geq x_{N}\left(1-\frac{K+1}{K-1}\left(\frac{1}{4}+K^{-1 /(2+\sqrt{6})}+K^{-2 /(2+\sqrt{6})}\right)\right) \\
& \geq 7 x_{N} / 100>0
\end{aligned}
$$

ce qui prouve (27), et donc que pour tout $N \geq 1, \mathcal{A}_{N}$ est admissible.

Nous devons montrer maintenant que l'ensemble $\mathcal{A}=\bigcup_{N=1}^{\infty} \mathcal{A}_{N}=\left\{a_{1}<\right.$ $\left.a_{2}<\ldots\right\}$ est aussi admissible. Raisonnons par l'absurde, et supposons que $\mathcal{A}$ a deux sous-ensembles de mêmes sommes, avec un nombre différent d'éléments, c'est-à-dire qu'il existe des entiers $i_{1}<i_{2}<\ldots<i_{u}, j_{1}<j_{2}<$ $\ldots<j_{v}$ tels que

$$
\sum_{k=1}^{u} a_{i_{k}}=\sum_{k=1}^{v} a_{j_{k}}, u \neq v .
$$

Nous pouvons supposer que $i_{u} \leq j_{v}$, et que $a_{i_{1}}, \ldots, a_{i_{n}}, a_{j_{1}}, \ldots, a_{j_{n}}$ (avec $u \neq v$ ) est une solution telle que $\bar{j}_{v}=\max \left(i_{u}, j_{v}\right)$ est minimal. On définit $N$ par $a_{j_{n}} \in \mathcal{A}_{N}$ (on a évidemment $N \geq 2$ ) et soit $u^{\prime}$ et $v^{\prime}$ les plus grands nombres tels que

$$
\left\{a_{i_{1}}, a_{i_{2}}, \ldots, a_{i_{u^{\prime}}}\right\} \subset \bigcup_{n=1}^{N-1} \mathcal{A}_{n}
$$

(et l'on a, ou bien $u^{\prime}=u$, ou bien $a_{i_{u^{\prime}+1}} \in \mathcal{A}_{N}$ ) et

$$
\left\{a_{j_{1}}, a_{j_{2}}, \ldots, a_{j_{n}}\right\} \subset \bigcup_{n=1}^{N-1} \mathcal{A}_{n}
$$

si l'on a $a_{i_{1}} \in \mathcal{A}_{N}$, on pose $u^{\prime}=0$. Alors $T_{n} \mid a_{i_{k}}$ pour $u^{\prime}<k \leq u$ et $T_{N} \mid a_{j_{k}}$ pour $v^{\prime}<k \leq v$, et (30) entraîne

$$
\sum_{k=1}^{u^{\prime}} a_{i_{k}} \equiv \sum_{k=1}^{v^{\prime}} a_{j_{k}} \bmod T_{N} .
$$


De plus, nous avons

$$
\left|\sum_{k=1}^{n^{\prime}} a_{i_{k}}-\sum_{k=1}^{v^{\prime}} a_{j_{k}}\right| \leq \sum_{n=1}^{N-1} \sum_{a \in \mathcal{A}_{n}} a<T_{N} .
$$

Par (31) et (32), il vient

$$
\sum_{k=1}^{n^{\prime}} a_{i_{k}}=\sum_{k=1}^{v^{\prime}} a_{j_{k}}
$$

et tous les termes ci-dessus appartiennent à $\bigcup_{n=1}^{N-1} \mathcal{A}_{n}$. Mais $j_{v}$ a été choisi minimum ; cela implique

$$
u^{\prime}=v^{\prime}
$$

On a ensuite par (30), (33) et (34)

$$
\sum_{k=u^{\prime}+1}^{u} a_{i_{k}}=\sum_{k=v^{\prime}+1}^{v} a_{i_{k}}
$$

et les nombres de termes dans les deux sommes ci-dessus sont différents : $u-u^{\prime} \neq v-v^{\prime}$. De plus tous ces termes appartiennent à $\mathcal{A}_{N}$, mais ceci est impossible puisque nous avons démontré que $\mathcal{A}_{N}$ est admissible. Cette contradiction termine la preuve que $\mathcal{A}$ est admissible.

Il reste à démontrer (20). Pour $x>x_{1}$, on définit $N$ par $x_{N-1}<x \leq x_{N}$. D'après la définition $\operatorname{de} \mathcal{A}$, nous avons

$$
\begin{aligned}
& A(x) \geq A\left(x_{N-1}\right) \geq\left|\mathcal{A}_{N-1}\right|=\left[x_{N-1}^{(\sqrt{6}-2) / 2}\right]= \\
& \quad=\left[\left(x_{N}^{2 /(2+\sqrt{6})}\right)^{(\sqrt{6}-2) / 2}\right]=\left[x_{N}^{5-2 \sqrt{6}}\right]>x_{N}^{5-2 \sqrt{6}}-1
\end{aligned}
$$

et cela achève la démonstration du théorème 2 . 
Table numérique

\begin{tabular}{|c|c|c|c|c|c|}
\hline$N$ & $a(N)$ & $F(N)$ & $p(N)$ & $\begin{array}{l}\text { Nombre de } \\
\text { solutions } \\
\text { impaires } \\
\text { N-optimales }\end{array}$ & $h(N)$ \\
\hline 1 & 1 & 1 & 1 & & 1 \\
\hline 2 & 3 & 2 & 1 & & 1 \\
\hline 3 & 6 & 2 & 2 & 1 & 2 \\
\hline 4 & 12 & 3 & 2 & & 1 \\
\hline 5 & 21 & 3 & 4 & 1 & 1 \\
\hline 6 & 36 & 4 & 1 & & 3 \\
\hline 7 & 59 & 4 & 4 & 1 & 2 \\
\hline 8 & 98 & 4 & 13 & & 1 \\
\hline 9 & 152 & 5 & 1 & & 5 \\
\hline 10 & 241 & 5 & 5 & & 2 \\
\hline 11 & 362 & 5 & 10 & 2 & 3 \\
\hline 12 & 545 & 6 & 1 & & 7 \\
\hline 13 & 794 & 6 & 2 & & 7 \\
\hline 14 & 1165 & 6 & 5 & & 7 \\
\hline 15 & 1650 & 6 & 13 & 2 & 6 \\
\hline 16 & 2378 & 7 & 1 & & 10 \\
\hline 17 & 3315 & 7 & 2 & & 10 \\
\hline 18 & 4621 & 7 & 6 & & 10 \\
\hline 19 & 6335 & 7 & 14 & 2 & 10 \\
\hline 20 & 8742 & 8 & 1 & & 13 \\
\hline 21 & 11688 & 8 & 2 & & 13 \\
\hline 22 & 15792 & 8 & 5 & & 13 \\
\hline 23 & 20918 & 8 & 11 & 1 & 13 \\
\hline 24 & 27713 & 8 & 20 & & 13 \\
\hline 25 & 36260 & 9 & 1 & & 17 \\
\hline
\end{tabular}




\begin{tabular}{|c|c|c|c|c|c|}
\hline$N$ & $a(N)$ & $F(N)$ & $p(N)$ & $\begin{array}{c}\text { Nombre de } \\
\text { solutions } \\
\text { impaires } \\
\text { N-optimales }\end{array}$ & $h(N)$ \\
\hline 26 & 47479 & 9 & 2 & & 17 \\
\hline 27 & 61065 & 9 & 5 & & 17 \\
\hline 28 & 79139 & 9 & 11 & & 17 \\
\hline 29 & 101145 & 9 & 23 & 2 & 17 \\
\hline 30 & 128998 & 10 & 1 & & 21 \\
\hline 31 & 163421 & 10 & 2 & & 21 \\
\hline 32 & 207304 & 10 & 5 & & 21 \\
\hline 33 & 259298 & 10 & 10 & & 21 \\
\hline 34 & 325692 & 10 & 20 & & 21 \\
\hline 35 & 405614 & 10 & 38 & 2 & 21 \\
\hline 36 & 503625 & 11 & 1 & & 26 \\
\hline 37 & 622853 & 11 & 2 & & 26 \\
\hline 38 & 769232 & 11 & 5 & & 26 \\
\hline 39 & 941482 & 11 & 10 & & 26 \\
\hline 40 & 1156143 & 11 & 21 & & 26 \\
\hline 41 & 1410171 & 11 & 39 & 2 & 26 \\
\hline 42 & 1711663 & 12 & 1 & & 31 \\
\hline 43 & 2076250 & 12 & 2 & & 31 \\
\hline 44 & 2513965 & 12 & 5 & & 31 \\
\hline 45 & 3023894 & 12 & 10 & & 31 \\
\hline 46 & 3639397 & 12 & 20 & & 31 \\
\hline 47 & 4366367 & 12 & 37 & 1 & 31 \\
\hline 48 & 5212730 & 12 & 65 & & 31 \\
\hline 49 & 6225734 & 13 & 1 & & 37 \\
\hline 50 & 7413601 & 13 & 2 & & 37 \\
\hline
\end{tabular}

[1] P. ERDÖS, Számelméleti megjegyzések, III, Mat. Lapok 13 (1962), 28-38.

[2] E.G. STRAUS, On a problem in combinatorial number theory, J. Math. Sci. I (1966), 77-80.

P. Erdös, A. Sárközy

Magyar Tudományos Akadémia 
Matematikai Kutató Intézete

Realtanoda utca 13-15

PF 127

H-1364 BUDAPEST V - HONGRIE.

et

J.-L. NICOLAS

Departement de Mathématiques, bât. 101

Université Claude Bernard, Lyon 1

F-69622 VILLEURBANNE Cedex - FRANCE. 